\section{Was there 26-Myr periodicity of extinctions?}

HOFFMAN $^{1}$ has questioned our claim ${ }^{2}$ that extinction events during the past $250 \mathrm{Myr}$ exhibit a 26-Myr periodicity. Hoffman argues that the apparent periodicity originates not from any evolutionary signal but from arbitrariness in the choice of taxonomic level, treatment of data and selection of geologic timescales in our analysis. His letter succeeds in outlining many problems inherent in working with data from the fossil record. However, we find his conclusion unconvincing because there is no hypothesis-testing. In fact, when statistical tests and independent criteria are applied, we find that Hoffman's results support our contention of periodicity better than our initial analyses.

In our initial paper ${ }^{2}$ we analysed a set of data on fossil marine families that was highly culled in order to enhance the signal-to-noise ratio. Hoffman implies that this culling may have induced an appearance of periodicity. However, our subsequent analyses ${ }^{3,4}$ indicate that this culling had no important consequences and an evident 26-Myr periodicity remains when a variety of extinction metrics (including rate and probability of extinction) are computed from the complete, unculled data set. Indeed, Hoffman's own measures of extinction intensity, summarized in his Table 1, also show this if consistent patterns rather than random noise are sought. Ten of Hoffman's 43 stages exhibit peaks (local maxima) in more than half of the 21 metrics (including the unillustrated regression residuals for number of family extinctions per stage). The ten stages are the Guadalupian, Olenekian, Norian, Pliensbachian, Bajocian, Tithonian, Cenomanian, Maestrichtian, late Eocene and middle Miocene. Of these, all but the Olenekian, Bajocian and middle Miocene have been independently recognized as containing extinction events by palaeontologists using detailed biostratigraphic data on species and genera ${ }^{3,5}$, and only one other stage (the Pliocene) in the Guadalupianto-Recent interval has been so recognized. The probability that the family data would encompass 7 of 8 events in 10 random peaks among 43 stages is $<0.00003$, strongly indicating that the consistent peaks among Hoffman's metrics result from evolutionary variation rather than artefact. This conclusion is further supported by the good correlation between familial and generic ${ }^{4,6}$ extinctions throughout the 43 stages. (For per cent extinction, $r=0.893$ and for probability of extinction $r=0.877$.)

Statistical tests similar to those used in our initial paper indicate that the 10 stages listed above exhibit a significant $(p<$ 0.002 ) nonrandomness at a $26-\mathrm{Myr}$ perio- dicity. Tests of this periodicity result in a standard deviation of differences between observed and expected peaks of $3.28 \mathrm{Myr}$ using the Harland et al. timescale ${ }^{7}$ and 5.53 Myr using the Odin timescale ${ }^{8}$; both fits are better than those obtained in our original analysis ${ }^{2}$. Fits with even higher significance are obtained if only the four most recent, and therefore most reliably dated $^{9}$, stages are tested ${ }^{4}$.

Why did Hoffman conclude that the familial data appeared random with respect to extinction? It may have been because he used a necessary but not sufficient criterion for randomness. $\mathrm{He}$ argued that if random, the extinction metrics should display an average frequency of one peak in four stages; because our data displayed this frequency, he concluded that they were random. But there is a great difference between an average frequency of occurrence and a regular occurrence. A fair coin will, on average, come up heads once in two flips, but if it comes up heads regularly every other flip, we should suspect its fairness. In our analysis of the extinction data, our randomization test ${ }^{2}$ actually converted the data into random walks with the same frequency of maxima as in the observed time series. Yet, when compared to these random walks, we found that the observed distribution of maxima was significantly $(p<0.01)$ more uniform than in the random walks. Thus, we rejected the hypothesis of randomness in favour of periodicity. We suggest that Hoffman might have done the same if he had performed the proper statistical tests.

\section{J. JOHN SEPKOSKI JR DAVID M. RAUP}

Department of the Geophysical Sciences, University of Chicago,

5734 S. Ellis Avenue,

Chicago, Illinois 60637, USA

1. Hoffman, A. Nature 315, 659-662 (1985)

2. Raup, D. M. \& Sepkoski, J. J. Jr Proc. natn. Acad. Sci U.S.A. 81, 801-805 (1984)

3. Sepkoski, J. J. Jr \& Raup, D. M. in Dynamics of Extinction (ed. Elliott, D.) 3-36 (Wiley, New York, 1986).

4. Raup, D. M. \& Sepkoski, J. J. Jr Science 231, 833-836 1986)

5. Sepkoski, J. J. Jr Spec. Pap. geol. Soc. Am. 190, 283-289 (1982)

6. Sepkoski, J. J. Jr in Patterns and Process in the History of Life (eds Raup, D. M. \& Jablonski, D.) (Springer, Berlin, in the press).

7. Harland, W. B. et al. A Geologic Time Scale (Cambridge University Press, 1983).

8. Odin, G. S. (ed.) Numerical Dating in Stratigraphy (Wiley, New York, 1982).

9. Maddox, J. Nature 315, 627 (1985)

HOFFMAN $^{1}$ and Hoffman and Ghiold ${ }^{2}$ recently presented a statistical argument that has been widely regarded ${ }^{3-5}$ as casting serious doubt upon Raup and Sepkoski's ${ }^{6}$ proposed 26-Myr extinction periodicity. Here I show that their conclusion is false and reflects an incorrect application of their own neutral model. When properly applied, the model actually supports Raup

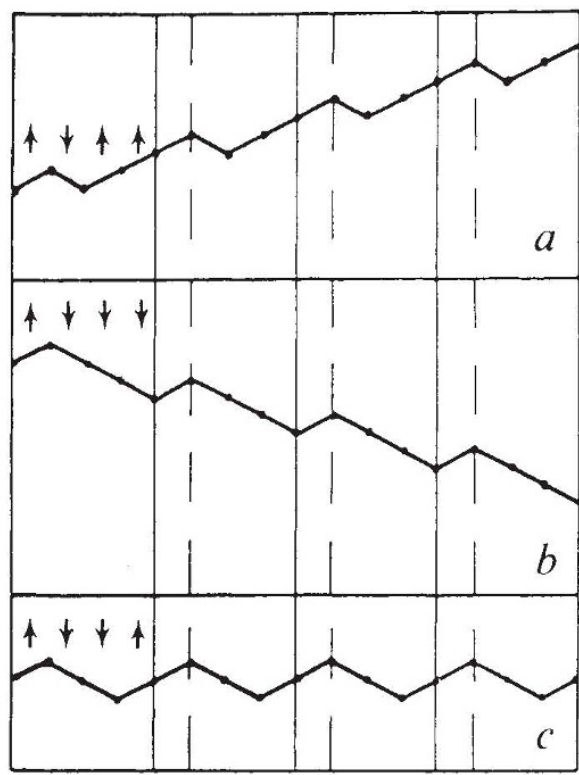

Fig. 1 The leftmost column shows the three ways of producing exactly one local extinction maximum in the second of a sequence of 4 stages. These are the unit sequences referred to in the text. Arrows indicate the direction of change (increase or decrease) in the extinction metric in each unit sequence. The unit sequences are repeated, producing three long periodic sequences of period 4 . Although $a, b$ and $c$ each involve repetition of a single unit sequence, any unit sequence may follow any other and still create a periodic series. The magnitudes of the

changes in extinction metric are ignored.

and Sepkoski's position and underscores the need for a causal explanation.

Hoffman and Hoffman and Ghiold's argument is as follows:

(1) Given Raup and Sepkoski's definition of mass extinction as any local maximum of extinction metric; that is, any increase followed by decrease, and

(2) Given that the probabilities of increase and decrease in extinction metric both equal 0.5 , and

(3) Given that the average stage length in the late Phanerozoic is $6.4 \mathrm{Myr}$, then the following conclusions hold:

(i) The probability that any particular stage is a local extinction maximum is equal to $(0.5)^{2}=0.25$.

(ii) Local extinction maxima are to be expected, on average, every four stages or every $4 \times 6.4=25.6 \mathrm{Myr}$.

(iii) "The appearance of approximately 26 million year periodicity is inevitable." 2

Conclusions $\mathrm{i}$ and ii are true. However, conclusion iii does not follow from premises 1-3 above and is false. The mistake is clarified by the following: Conclusion ii in more precise language, means that in a large number of samples of four stages, the arithmetic mean number of mass extinctions per sample will be 1 . However, for an extinction pattern to be periodic with a period of four stages requires that every fourth and only every fourth stage 